RECENT LABORATORY WORK ON MOLECULES OF POSSIBLE IMPORTANCE FOR INTERSTELLAR STUDIES

G. Herzberg

National Research Council of Canada

ottawa, Ontario, KIA OR6

Canada

One of the aims of our work at the National Research Council (ottawa) during the last thirty years has been to contribute laboratory data that are of possible astronomical interest especially for the study of the interstellar medium.

\title{
NEUTRAL MOLECULES
}

The detection of the first two interstellar molecules, $\mathrm{CH}$ and $\mathrm{CN}$ in the near ultraviolet region, needed no special laboratory work since all the information for this identification had been assembled a long time before. As is well known, the radio-frequency detection of $\mathrm{CH}$ presented considerable difficulty. Various attempts to determine the $\Lambda$-type doubling in the $\mathrm{CH}$ ground state, $\mathrm{X}^{2} \mathrm{I}$, from the optical spectrum were required (Douglas and Elliott (1965), Goss (1966), Baird and Bredohl (1971)) before this transition was observed by Rydbeck et al. (1974). Even now the radio lines of $\mathrm{CH}$ have not been observed in the laboratory.

A considerable amount of work has been done at NRC on the spectra of $\mathrm{H}_{2}, \mathrm{HD}$ and $\mathrm{D}_{2}$. The earlier work on these molecules, with the exception of that of Wilkinson (1968), was marred by the lack of good standards. This was not a difficulty for the new laboratory measurements of HD (Dabrowski and Herzberg (1976)) and as a result the agreement between the Copernicus observations and the laboratory spectra is now excellent.

For the detection of diatomic carbon, that is, the $\mathrm{C}_{2}$ molecule, it was first necessary to establish the nature of the ground state of this molecule. This was done in the laboratory work of Ballik and Ramsay (1963) who established that the ground state is ${ }^{1} \sum_{g}{ }^{+}$. In Figure 1 the known 


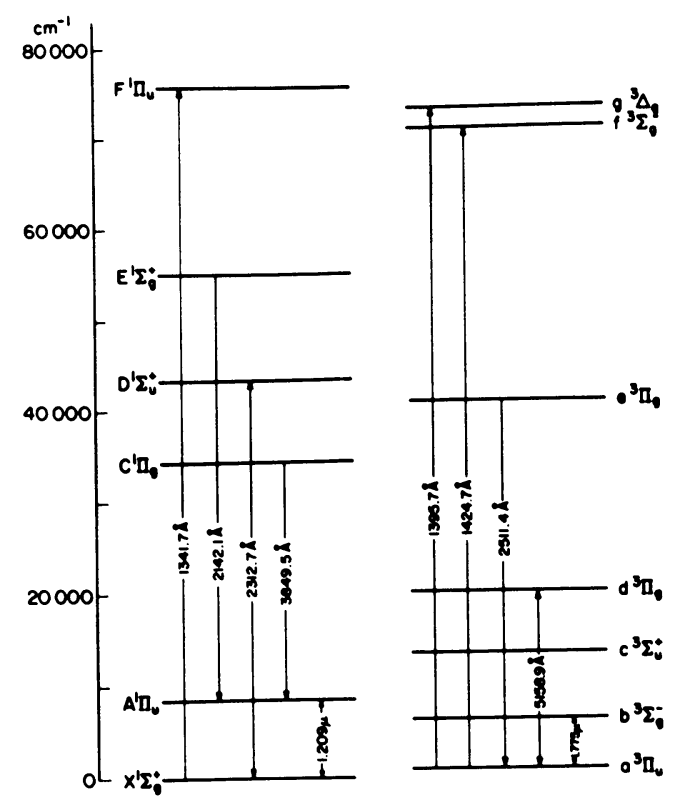

Fig.1. Electronic energy levels and observed transitions in $\mathrm{C}_{2}$

electronic states of $\mathrm{C}_{2}$ are presented. It was only last year that the transition from the ground state to the lowest excited state (the Phillips bands) was observed in interstellar absorption by Chaffee and Lutz (1978). At the same time Snow (1978) observed in the ultraviolet the Mulliken bands in absorption.

It is surprising that the $\mathrm{OH}$ molecule, which was the first molecule identified by radio-astronomy in interstellar space, was only quite recently observed optically, first in the vacuum ultraviolet by Snow (1976) using the 1222 A band found in the laboratory somewhat earlier by Douglas (1974) and still more recently in the ordinary ultraviolet near $3080 \AA$ by Crutcher and Watson (1976).

In the 1950's the optical spectra of HCO and HNO were discovered at NRC (Herzberg and Ramsay 1955, Dalby 1958). Subsequently microwave spectra were observed in the laboratory by Saito (1972) and Saito and Takagi (1973) and on that basis these two radicals were detected in the interstellar medium by Snyder et al. (1976) and Ulich et al. (1977). It is somewhat surprising to find HCO present in spite of the fact that its dissociation energy is rather small, of the order of $l \mathrm{eV}$. It is of interest to mention that $\mathrm{HCO}$ in the visible region (near 5400, 5900 and 6460 \&) has diffuse absorption bands (in addition to sharp ones). 
The $R(0)$ lines of these bands should be observable in the interstellar medium if sufficient concentrations of HCO are present. However they do not agree with the known diffuse interstellar absorption features.

The spectrum of the $\mathrm{NH}_{2}$ radical in the optical region was discovered in 1952 (Herzberg and Ramsay) and was analysed by Dressler and Ramsay in 1959. The laboratory work on this spectrum is continuing (Johns, Ramsay and Ross 1976). While some microwave lines have been found in the laboratory by microwave optical double resonance and laser magnetic resonance no features have yet been

identified in the interstellar medium. The optical spectrum of $\mathrm{NH}_{2}$ is, however, a prominent feature in the spectra of the heads of comets.

The $\mathrm{CH}_{2}$ radical has been found by laboratory experiments to have a triplet ground state $\left({ }^{3} \mathrm{~B}_{1}\right)$ and a number of lowlying singlet states (Herzberg 1961, Herzberg and Johns 1971). The optical absorption spectrum lies in the vacuum ultraviolet near $1415 \AA$. In $\mathrm{CH}_{2}$, unlike $\mathrm{CD}_{2}$, the individual rotational lines of the absorption band are fairly diffuse. The $R(0)$ and $P(I)$ lines lie at 1415.50 and $1416.05 \AA$. It is perhaps significant that Snow, York and Resnick (1977) report in a number of stars as the only diffuse feature in the vacuum ultraviolet one somewhat questionable line at $1416 \AA$.

The $\mathrm{CH}_{3}$ radical (Herzberg 1961) has diffuse absorption bands with peaks at 2157.6,2163.6, 1502.4 and 1496.89 $\AA$. It seems likely that they will eventually be found in interstellar absorption.

Recently we have found (Herzberg 1979) in hollow cathode discharges in hydrogen a number of emission bands which have been definitely assigned as belonging to the Rydberg spectrum of triatomic hydrogen $\left(\mathrm{H}_{3}\right.$ and $\left.\mathrm{D}_{3}\right)$. All rotational lines are diffuse, for $\mathrm{H}_{3}$ much more so than for $\mathrm{D}_{3}$. The observed intensity alternation confirms the $\mathrm{D}_{3 \mathrm{~h}}$ symmetry of this molecule, i.e. that the nuclei form an equilateral triangle with a $\mathrm{H}-\mathrm{H}$ distance of $0.87 \mathrm{~A}$ as in $\mathrm{H}_{3}{ }^{+}$. The new spectra are emitted in the process of dissociative recombination of $\mathrm{H}_{3}{ }^{+}$and $\mathrm{D}_{3}+{ }^{+}$. This process has been assumed to be the cause of the eventual destruction of $\mathrm{H}_{3}{ }^{+}$in the interstellar medium. It seems possible therefore that the $\mathrm{H}_{3}$ spectrum will be observable in astronomical spectra. 
MOLECULAR IONS

Recently Alberti and Douglas (1975) have made new measurements of the $\mathrm{NO}^{+}$spectrum, first discovered by Baer and Miescher (1953), with the principal aim of having accurate predictions for possible identification of interstellar lines of this ion. Table I summarizes their

Table I. $\mathrm{NO}^{+}$lines of astronomical interest

\begin{tabular}{c|c}
\hline Transition & $R(0)$ \\
\hline${ }^{1} \Pi(v=0)-{ }^{1} \sum(v=0)$ & 1368.2404 \\
${ }^{1} \Pi(v=1)-{ }^{1} \sum(v=0)$ & 1339.6361 \\
${ }^{1} \Pi(v=2)-{ }^{1} \sum(v=0)$ & 1312.9400 \\
${ }^{1} \Pi(v=3)-{ }^{1} \sum(v=0)$ & 1288.0254 \\
${ }^{1} \Pi(v=4)-{ }^{1} \sum(v=0)$ & 1264.7900 \\
${ }^{1} \sum(v=I)-{ }^{1} \sum(v=0)$ & $2347.74\left(\mathrm{~cm}^{-1}\right)$ \\
${ }^{1} \sum(J=I-J=0)$ & $119187 \pm 30 \mathrm{MHz}^{1}(\mathrm{JH}=$ \\
\hline
\end{tabular}

results. Colbourn and Douglas (1977) made new measurements of $\mathrm{N}_{2}{ }^{+}$and $\mathrm{O}_{2}{ }^{+}$with the same aim. It would seem worthwhile to make an all-out attempt to observe the $\mathrm{N}_{2}{ }^{+}$spectrum since the $\mathrm{N}_{2}{ }^{+}$lines have a high $f$ value and are very conveniently located in the spectrum (3914 \&).

Ms. Dabrowski and I (1977) have used the theoretical potential function of $\mathrm{HeH}^{+}$derived by Wolniewicz (1965) and Kolos and Peek (1976) in order to predict the infrared spectrum of this ion. We have also calculated the quasibound levels of this ion which lie above the dissociation limit at $1.845 \mathrm{eV}$. The inverse predissociation via these quasibound levels may lead to a recombination spectrum that has also been predicted. Attempts in this laboratory to observe the infrared spectrum of $\mathrm{HeH}^{+}$have so far been unsuccessul.

Discharges through He-Ne mixtures yield two features near $4200 \AA$ and similar spectra occur near $1400 \AA$ in $\mathrm{Ar}-\mathrm{He}$ mixtures. Tanaka, Yoshino and Freeman (1975) first correctly recognized the nature of the transitions responsible for these spectra. They are charge transfer spectra in which the molecule goes from a state that is essentially $\mathrm{He}^{+}+\mathrm{Ne}$ to a lower state that is essentially $\mathrm{He}+\mathrm{Ne}^{+}$and similarly for HeAr ${ }^{+}$. Figure 2 gives a potential energy diagram for $\mathrm{HeNe}^{+}$based on a fairly complete analysis of the spectrum near $4200 \AA$ (Dabrowski and Herzberg 1978). The ground state of HeNe ${ }^{+}$has a fairly large dissociation energy $(0.6 \mathrm{eV})$. On account of the 


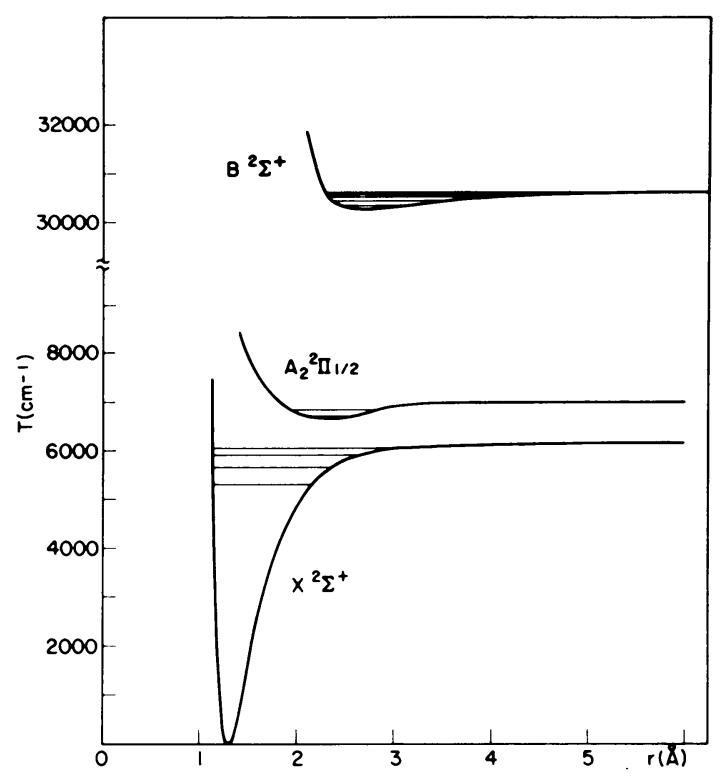

Fig.2. Potential energy functions of $\mathrm{HeNe}^{+}$

Franck-Condon principle we were unable to observe the lowest vibrational levels of the ground state but were able to establish the vibrational numbering on the basis of isotope shifts in ${ }^{3} \mathrm{HeNe}^{+}$. In the interstellar medium it seems likely that $\mathrm{HeNe}^{+}$if once formed will reach by infrared transition the lowest level of the ground state. A microwave line at $173550 \mathrm{MHz}$ and an infrared line at $1280 \mathrm{~cm}^{-1}$ are predicted.

As is well known, the ions $\mathrm{HCO}^{+}$and $\mathrm{N}_{2} \mathrm{H}^{+}$were first observed by radio-astronomical methods before they were observed in the laboratory by Woods et al. (1975) and Saykally et al. (1976) in absorption in electric discharges through suitable gas mixtures. We have attempted, so far unsuccessfully, to observe the ultraviolet spectra of these ions. It seems possible that some of the unidentified interstellar lines in the vacuum ultraviolet might be due to the electronic transition of these molecular ions.

An electronic transition of $\mathrm{H}_{2} \mathrm{O}^{+}$, very similar to that of $\mathrm{NH}_{2}$, was first observed by H. Lew (1973, 1976) in emission of hot cathode discharges. In the upper state the ion is essentially linear while in the lower state it is strongly bent with an angle of about $110^{\circ}$. The molecular constants of $\mathrm{H}_{2} \mathrm{O}^{+}$may be found in Dr. Lew's paper. In Table II some of the predicted interstellar lines are listed. None have yet been found. 
Table II. Predicted interstellar lines of $\mathrm{H}_{2} \mathrm{O}^{+}$

a) in the microwave region $(b)$ in the visible region

\begin{tabular}{|c|c|c|c|c|}
\hline $\begin{array}{c}\text { Rotational } \\
\text { transition }\end{array}$ & $\begin{array}{c}\text { Spin } \\
\text { component }\end{array}$ & $\begin{array}{c}\text { Frcquency } \\
\text { (GHz) }\end{array}$ & Transition & $\lambda_{1 \mathrm{ab}}(\AA)$ \\
\hline $220-313$ & $\begin{array}{l}F_{1} \\
F_{2}\end{array}$ & $\begin{array}{l}144.44 \pm 0.39 \\
187.19 \pm 0.42\end{array}$ & \multirow{6}{*}{$\begin{array}{l}(0,6,0)-(0,0,0) \\
(0,8,0)-(0,0,0) \\
(0,10,0)-(0,0,0) \\
(0,12,0)-(0,0,0) \\
(0,14,0)-(0,0,0)\end{array}$} & \multirow{6}{*}{$\begin{array}{l}6973 \cdot 720 \\
6970 \cdot 152 \\
6146.802 \\
6147 \cdot 375 \\
5478.548 \\
5481.217 \\
4922 \cdot 382 \\
4917.854 \\
4469 \cdot 358 \\
4468.823\end{array}$} \\
\hline $4_{14}-3_{21}$ & $\begin{array}{l}F_{1} \\
F_{2}\end{array}$ & $\begin{array}{l}134.88 \pm 0.40 \\
103.87 \pm 0.39\end{array}$ & & \\
\hline $3_{30}-4_{23}$ & $\begin{array}{l}F_{1} \\
F_{2}\end{array}$ & $\begin{array}{l}218.61 \pm 0.48 \\
266.56 \pm 0.50\end{array}$ & & \\
\hline $422-332$ & $\begin{array}{l}F_{1} \\
F_{2}\end{array}$ & $\begin{array}{r}34.27 \pm 0.54 \\
-13.37 \pm 0.54\end{array}$ & & \\
\hline$\sigma_{33}-5_{42}$ & $\begin{array}{l}F_{1} \\
F_{2}\end{array}$ & $\begin{array}{l}278.89 \pm 0.72 \\
234.41 \pm 0.72\end{array}$ & & \\
\hline$\sigma_{52}-7_{43}$ & $\begin{array}{l}F_{1} \\
F_{2}\end{array}$ & $\begin{array}{r}64.07 \pm 1.6 \\
105.63 \pm 1.6\end{array}$ & & \\
\hline
\end{tabular}

From the photoelectron spectrum of $\mathrm{NH}_{3}$ it is readily seen that there is an excited state of $\mathrm{NH}_{3}{ }^{+}$at $4.77 \mathrm{eV}$ above the $\mathrm{X}^{2} \mathrm{~A}_{2}$ " ground state (assuming $\mathrm{D} 3 \mathrm{~h}$ symmetry). Therefore an emission spectrum near $2300 \AA$ may be expected. Since the excited state is in all probability a ${ }^{2} \mathrm{E}^{\prime}$ state the transition between it and the ground state is electronically forbidden. However, vibronic interaction will make some of the vibrational transitions in the forbidden electronic transition allowed provided that either in the upper or lower state a degenerate vibration is excited. Using electron bombardment excitation of a $\mathrm{NH}_{3}$ jet we have observed a number of weak but distinct features in the 2400-2200 $\mathrm{A}$ region. One simple band near $2338 \AA$ appears in which apparently alternate lines are missing. If this were true it would prove that the band is a sub-band of a $\perp$ band in a planar molecule with $\mathrm{D}_{3 \mathrm{~h}}$ symmetry as expected for $\mathrm{NH}_{3}{ }^{7}$.

The ion-molecule reaction

$$
\mathrm{H}_{2}{ }^{+}+\mathrm{H}_{2} \rightarrow \mathrm{H}_{3}^{+}+\mathrm{H}+1.75 \mathrm{eV}
$$

is one of the fastest known reactions. As a result one finds that in every discharge through $\mathrm{H}_{2}$ the $\mathrm{H}_{3}{ }^{+}$ion is the most abundant ion (except at very low pressure). It is believed that also in the interstellar medium $\mathrm{H}_{3}{ }^{+}$is $a$ fairly abundant ion. It seems to play an important role in the processes that lead to molecule formation (see Herbst and Klemperer 1973, Watson 1973, Suzuki 1979). 
In order to detect $\mathrm{H}_{3}{ }^{+}$spectroscopically one is dependent on its infrared bands since according to theoretical calculations all excited electronic states seem to be unstable. Up to now the infrared spectrum has not been observed in the laboratory. However, H. Lew, $\mathrm{J}$. Sloan and I at ottawa have made a number of attempts to observe this spectrum in emission from a hollow cathode discharge cooled to liquid nitrogen temperature. Using a Fourier transform spectrometer we have observed a number of infrared lines in the region between 5000 and $2000 \mathrm{~cm}^{-1}$. Several of these lines are known to be due to $\mathrm{H}_{2}$ (transitions between various excited electronic states) but a good number of the lines are unidentified. In order to ascertain whether some of these unidentified lines are due to $\mathrm{H}_{3}{ }^{+}$one must either have reliable predictions or differentiate by discharge conditions or by electromagnetic deflection. We find that some of the unidentified lines are much weaker in the anode glow than in the cathode glow. Three years ago Carney and Porter (1976) made some predictions about the theoretically expected infrared spectrum. We find that the strongest predicted lines agree with those observed infrared lines that show considerable difference between the cathode and anode glow. It would be hasty to consider this as proof of the observation of the spectrum of $\mathrm{H}_{3}{ }^{+}$since there are quite a number of $\mathrm{H}_{2}$ lines also. We either have to observe complete bands allowing the use of the method of combination differences for identification or we have to look at an electronically deflected beam of $\mathrm{H}_{3}+$ ions. Such experiments are now in preparation.

\section{References}

Alberti, F. and Douglas, A.E.: 1975, Can. J. Phys. 53, 1179. Baer, P. and Miescher, E.: 1953, Helv. Phys. Acta $\frac{26}{6}, 91$. Baird, K.M. and Bredohl, H.: 1971, Astrophys. J. 169, L83. Ballik, E.A. and Ramsay, D.A.: 1963, Astrophys. J. 137, 61. Carney, G.D. and Porter, R.N.: 1976, J. Chem. Phys. 65,3547 . Chaffee, F.H. Jr. and Lutz, B.L.: 1978, Astrophys. J. 221 , L91.

Colbourn, E.A. and Douglas, A.E.: 1977, J. Mol. Spectrosc. 65,332 .

Crutcher, R.M. and Watson, W.D.: 1976, Astrophys. J. 203, L123.

Dabrowski, I. and Herzberg, G.: 1976, Can. J. Phys. 54, 525. Dabrowski, I. and Herzberg, G.: 1977, Trans. N.Y. Acad. Sci. 38, II, 14 .

Dabrowski, I. and Herzberg. G.: 1978, J. Mol. Spectrosc.

Dalb $\frac{73}{\text { y, }}$. F.W.: 1958, Can. J. Phys. 36, 1336. 
Douglas, A.E.: 1974, Can. J. Phys. 52, 318.

Douglas, A.E. and Elliott, G.A.: 1965, Can. J. Phys. 43, 496. Dressler, K. and Ramsay, D.A.: 1959, Phil. Trans. $251 \bar{A}, 553$. Goss, W.M.: 1966, Astrophys. J. 145, 707 .

Herbst, E. and Klemperer, W.: 1973, Astrophys. J. 185, 505. Herzberg, G.: 1961, Proc. Roy. Soc. 262A, 291.

Herzberg, G.: 1979, J. Chem. Phys. 70, 4806.

Herzberg, G. and Johns, J.W.C.: 1971, J. Chem. Phys. 54, 2276. Herzberg, G. and Ramsay, D.A.: 1952, J. Chem. Phys. 20, 347 . Herzberg, G. and Ramsay, D.A.: 1955, Proc. Roy. Soc. 233 A, 34. Johns, J.W.C., Ramsay, D.A. and Ross, S.C.: 1976, Can. J. Phys. 54, 1804 .

Kolos, W. and Peek, J.M.: 1976, Chem. Phys. 12, 381. Lew, H.: 1976, Can. J. Phys. 54, 2028. Lew, H. and Heiber, I.: 1973, J. Chem. Phys. 58, 1246. Rydbeck, O.E.H., Elldér, J., Irvine, W.M., Sume, A. and Hjalmarson, $\AA .: 1974$, Astron. Astrophys. 33, 315, 34, 479. Saito, S.: 1972, Astrophys. J. 178, L95.

Saito, S. and Takagi, K.: 1973, J. Mol. Spectrosc. 47, 99. Saykally, R.J., Dixon, T.A., Anderson, T.G., Szanto, P.G. and Woods, R.C.: 1976, Astrophys. J. 205, L101. Snow, T.P. Jr.: 1976, Astrophys. J. $204, \overline{\mathrm{L} 127}$. Snow, T.P. Jr.: 1978, Astrophys. J. $\overline{220}$, L93.

Snow, T.P. Jr., York, D.G. and Resnick, M.: 1977, Publ. Astron. Soc. Pacific 89, 758.

Snyder, L.E., Hollis, J.M. Lovas, F.J. and Ulich, B.L.: 1976, Astrophys. J. 209, 67.

Suzuki, H.: 1979, Prog. Theor. Phys. 62, October.

Tanaka, Y., Yoshino, K. and Freeman, D.E.: 1975, J. Chem. Phys. 62, 4484 .

Ulich, B.L., Hollis, J.M., Snyder, L.E.: 1977, Astrophys. J. 217, L105.

Watson, W.D.: 1973, Astrophys. J. 183, L17, 188, 35.

Wilkinson, P.G.: 1968, Can. J. Phys. 46, $122 \frac{1}{5}$

Wolniewicz, L.: 1965 , J. Chem. Phys. 43, 1087 .

Woods, R.C., Dixon, T.A., Saykally, R.J. and Szanto, P.G.: 1975, Phys. Rev. Letters 35, 1269.

\section{DISCUSSION FOLLOWING HERZBERG}

Hollenbach: Although grain composition may vary from place to place, I would think the correlation of diffuse bands with $A_{v}$ more likely if the bands were caused by grains; molecular abundances generally are very sensitive to parameters such as gas density and radiation fields along the line of sight.

Herzberg: The references I have quoted, and several others, suggest considerable difficulty for that explanation. spectrum of $\mathrm{H}_{3}{ }^{+}$?

What type of $\mathrm{H}_{2}$ lines might be contaminating the infrared Herzberg: The $\mathrm{H}_{2}$ lines in question represent transitions between several excited states of $\mathrm{H}_{2}$, for example $\mathrm{E}-\mathrm{B}$. 